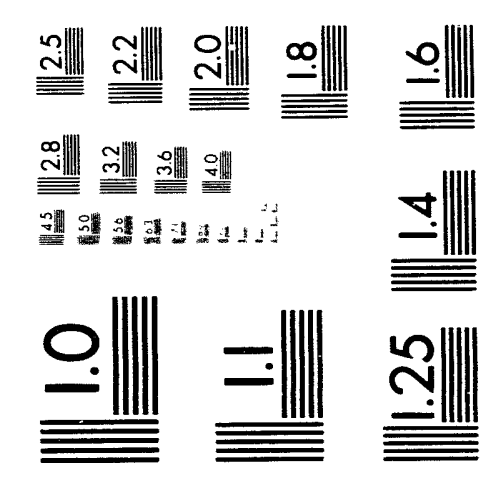



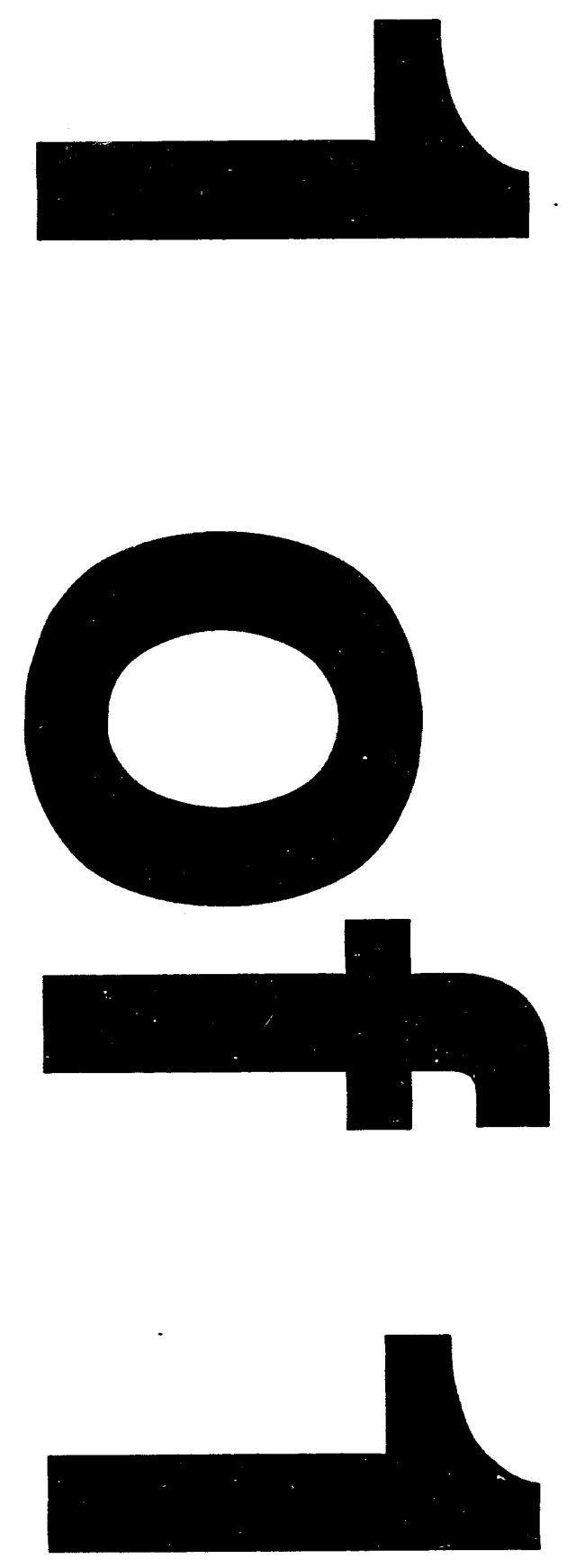


\section{LA-UR $93-3811$}

AUTHOR(S): H.-J. Ziock, K. Holzscheiter, A. Morgan, A.P.T. Palounek, J. Ellison, A.P. Heinson, M. Mason, S. J. Wimpenny, E. Barberis, N. Cartiglia, A. Gri11o, K. O'Shaughnessy, J. Rahn, P. Rinaldi, W.A. Rowe, H.F.-W. Sadrozinski, A. Seiden, E. Spencer, A. Webster, R. Wichmann, M. Wilder, M.A. Frautschi, J.A.J. Matthews, D. McDonald, D. Skinner, D. Coupal, and T. Pal

SUBMITTED TO: To be presented at IEEE Nuclear Science Symposium, November 3 , 1993

\section{DISCLAIMER}

This report was prepared as an account of work sponsored by an agency of the United States Government. Neither the United States Government nor any agency thereof, nor any of their employees, makes any warranty, express or implied, or assumes any legal liability or responsibility for the accuracy, completeness, or usefulness of any information, apparatus, product, or process disclosed, or represents that its use wiuld not infringe privately owned rights. Reference herein to any specific commercial product, process, or service by trade name, trademark, manufacturer, or otherwise does not necessarily constitute or imply its endorsement, recommendation, or favoring by the United States Government or any agency thereof. The views and opinions of authors expressed herein do not necessarily state or reflect those of the United States Government or any agency thereof.

By acceptance of this article. the putbisher recognizes that the US Government retains a nonexclusive. royalty-free license to publish or reproduce 


\title{
TEMPERATURE DEPENDENCE OF THE RADIATION INDUCED CHANGE OF DEPLETION VOLTAGE IN SILICON PIN DETECTORS
}

\author{
H.-J. Ziock, K. Holzscheiter, A. Morgan, and A.P.T. Palounek, \\ Los Alamos National Laboratory, Los Alamos, NM 87545
J. Ellison, A.P. Heinson, M. Mason, and S.J. Wimpenny, University of California, Riverside, CA 92521

\begin{abstract}
E. Barberis, N. Cartiglia, A. Grillo, K. O'Shaughnessy, J. Rahn, P. Rinaldi, W.A. Rowe, H.F-W. Sadrozinski, A. Seiden, E. Spencer, A. Webster, R. Wichmann, and M. Wilder, Santa Cruz Institute for Particle Physics, University of California, Santa Cruz, CA 95064

\author{
M.A. Frautschi, J.A.J. Matthews, D. McDonald, and D. Skinner, \\ University of New Mexico, Albuquerque, NM 87131 \\ D. Coupal, and T. Pal, \\ Superconducting Super Collider Laboratory, Dallas, TX 75237
}

\begin{abstract}
We present a study of how temperature affects the change in the depletion voltage of silicon PIN detectors damaged by radiation. We study the initial radiation damage and the shortterm and long-term annealing of that damage as a function of temperature in the range from $-10^{\circ} \mathrm{C}$ to $+50^{\circ} \mathrm{C}$, and as a function of $800 \mathrm{MeV}$ proton fluence up to $1.5 \times 10^{14} \mathrm{p} / \mathrm{cm}^{2}$. We express the pronounced temperature dependencies in a simple model in terms of two annealing time constants which depend exponentially on the temperature.
\end{abstract}

\section{INTRODUCTION}

The silicon microstrip detectors that will be used in the SDC experiment at the Superconducting Super Collider (SSC) will be exposed to very large fluences of charged particles, neutrons, and gammas. Over the projected 10-year life of the experiment, the total charged particle fluence is expected to be approximately $1.3 \times 10^{14} / \mathrm{cm}^{2}$ at a radial distance of $9 \mathrm{~cm}$ (the inner radius of the SDC silicon tracker), and $4.6 \times 10^{11} / \mathrm{cm}^{2}$ at $46.5 \mathrm{~cm}$ (the outer radius of the SDC silicon tracker). The comparable gamma fluence is of less importance. The neutron fluence will be dominated by backwards leakage out of the calorimeter and is expected to have a 10-year integral value of $2 \times 10^{13} / \mathrm{cm}^{2}[1]$. It should have a nearly uniform spatial distribution and an energy spectrum that is peaked at just under $1 \mathrm{MeV}$.

The charged particles arc all essentially minimum ionizing particles (MIPs) and cause both ionization and displacement damage in the bulk. A $1-\mathrm{MeV}$ neutron causes approximately the same amount of displacement damage as a MIP [2]. Based on that assessment, we chose to examine damage issues in silicon detectors using $800 \mathrm{MeV}$ protons, which are nearly minimum ionizing particles.
One of the efiects of displacement damage in silicon detectors is an increase in the leakage current of the detector [3], leading to increased shot noise and resistive heating. The effects of shot noise can be reduced by using pre-amplifiers with fast shaping times [4]. The exponential dependence of the leakage current on temperature [5] can lead to thermal runaway, which we have observed in detectors exposed to high fluences at high operating voltages.

Because of the strong temperature dependence of the leakage current, operating the detectors at reduced temperature $\left.(\sim)^{\circ} \mathrm{C}\right)$ solves both the shot noise and heating problems. However, low temperature operation requires understanding the temperature dependence of another bulk radiation effect: the change in effective dopant concentration and its annealing, which manifests itself as an increased depletion voltage at high hadron fluences. We have observed before [6] that the effective dopant concentration, as measured by the depletion voltage, varies as a function of fluence and of annealing time as shown in Figs. 1 and 2. Moreover, the data in Figs. 1 and 2 exhibit a pronounced temperature dependence, both during irradiation and during annealing. The goal of this study was to explain the fluence, time and temperature dependence of the change in depletion voltage by a simple model. That model then allows us to predict the behavior at large fluences and long times, and to select the optimal operating temperature.

\section{EXPERIMENT}

We irradiated silicon detectors with $800 \mathrm{MeV}$ and $647 \mathrm{MeV}$ proton beams at the Clinton P. Anderson Meson Physics Facility (LAMPF). The detectors received fluences of up to $1.5 \times 10^{14} \mathrm{p} / \mathrm{cm}^{2}$ over periods of approximately one week. Measurements of the leakage current I(V) and of the capacitance $\mathrm{C}(\mathrm{V})$ as functions of the bias voltage were made 


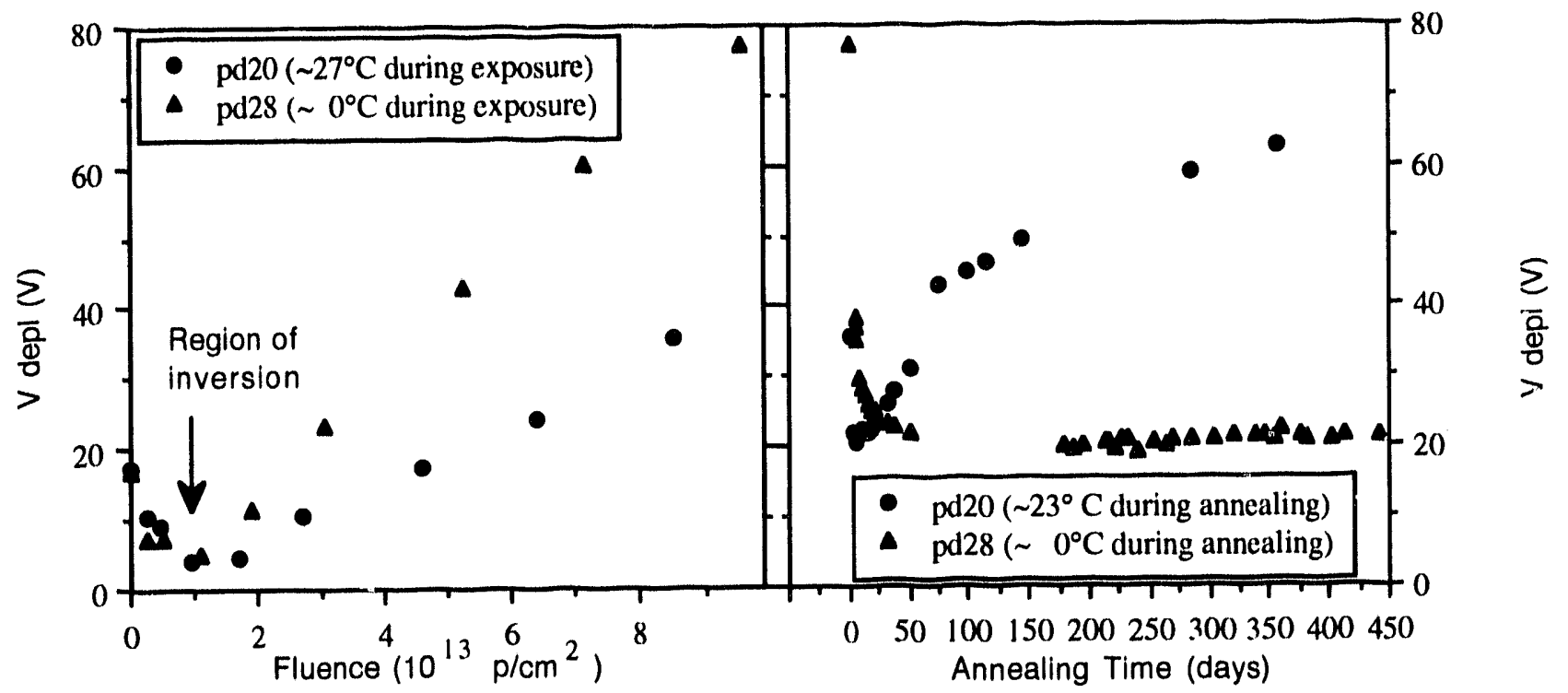

Figure 1): The depletion voltage of $170 \mu \mathrm{m}$ thick Hamamatsu photo-diodes as a function of fluence and as a function of annealing time for $0^{\circ} \mathrm{C}$ and room temperature operation. The last points on the left hand plot correspond to the first points on the right hand plot. From Ref. [6].

eight times during the runs. These measurements were made during few hour-long periods after the beam was temporarily turned off. Following the exposures, we continued the I(V) and the $C(V)$ measurements to the present, approximately one and two years for the two different exposures. We also have records from earlier irradiations with about four years of annealing time at room temperature.

The proton fluences were determined by activation measurements of aluminum foils placed on the individual detectors. Details are given in Ref. [6].

Two different detector types were irradiated: Hamamatsu PIN photo-diodes \#1723-06 with a depletion depth of $170 \mu \mathrm{m}$ [7]; and $300 \mu \mathrm{m}$ thick pad PIN devices manufactured by Micron Semiconductor [8]. In the presentation of the data, we have scaled up the depletion voltages of the photo-diodes by a factor $(300 / 170)^{2}$ ( $c f$. eq. 2 below), to account for the difference in thickness. All detectors were reverse-biased at $60 \mathrm{~V}$ or $80 \mathrm{~V}$ during irradiation. Some of the detectors were kept at ambient temperature, $21^{\circ} \mathrm{C}$ and $27^{\circ} \mathrm{C}$ during the two different exposures. A temperature sensing system that opened and closed a line supplying boil-off gas from a liquid nitrogen supply cooled the remaining devices to approximately $0^{\circ} \mathrm{C}$. For about one month following exposure to the bearn, the cold devices were stored in a freezer, while the ambient temperature devices remained at room termperature. Measurements of the depletion voltages of the devices continued during this period while small chambers were prepared to keep the devices at several different but fixed temperatures. Enough chambers were constructed to keep devices at $-10^{\circ} \mathrm{C}, 0^{\circ} \mathrm{C},+10^{\circ} \mathrm{C},+24^{\circ} \mathrm{C}^{\dagger}$,

\footnotetext{
† Some of the older " $24^{\circ} \mathrm{C}$ " devices kept were simply kept at
} ambient temperature. We use $24^{\circ} \mathrm{C}$ as a generic temperature to $+35^{\circ} \mathrm{C}$, and $+50^{\circ} \mathrm{C}$. The chambers were small, insulated boxes with a motor-driven muffin fan and a resistive heating element. A temperature sensor inside each box cycled power to the resistive heating element, maintaining the desired temperature within a range of about $\pm 0.5^{\circ} \mathrm{C}$. The fans ensured that no temperature gradients became established inside the boxes. They also served to dissipate any modest amounts of heat generated in the detector by I- $V$ heating when the detectors were biased. The sub-ambient temperature chambers vere placed inside a freezer set to $-20^{\circ} \mathrm{C}$.

Depletion voltages were determined by measuring the capacitance of the detectors as a function of the bias voltage using an HP 4284A capacitance meter at $10 \mathrm{kHz}$. We took the depletion voltage of a device to be the voltage at which a fit of the form:

$$
\mathrm{C}=\mathrm{a} \mathrm{V}^{\mathrm{n}}
$$

to the most steeply falling part of the data, intercepts a line of constant capacitance. The constant capacitance value was the full depletion capacitance of the devices prior to irradiation. This procedure was previously shown to give a reliable and frequency independent estimate of the depletion voltage [9].

\section{RESULTS}

\section{Initial Damage}

The rolation between the depletion voltage and the dopant concentration is linear and is given by:

$$
V_{D} \approx \frac{q d_{D}^{2}\left|N_{e f f}\right|}{2 \varepsilon_{s}}
$$

indicate both the controlled $24^{\circ} \mathrm{C}$ devices and the room temperature devices. 


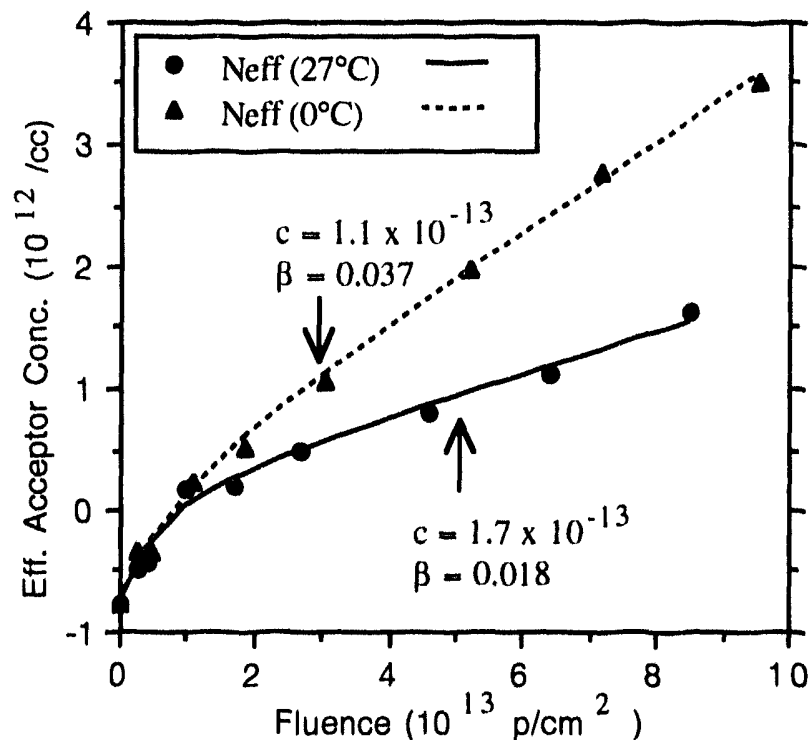

Figure 2): The dopant concentration of the devices shown in Fig. 1 as a function of fluence, and fits using eq. 3. From Ref. [6].

where $N_{\text {eff }}$ is the effective dopant concentration, $\varepsilon_{\mathrm{s}}$ is the permittivity of silicon, $q$ is the electron charge, and $d_{D}$ is the detector's full depletion depth. Fig. 2 shows the relation between the dopant concentration, as determined from the depletion voltage, and the proton fluence $(\phi)$. Each measurement was taken a few hours after the beam was temporarily turned off. We have shown before $[6,9]$ that the data in Fig. 2 can be explained by donor removal and acceptor creation, expressed as:

$$
\mathrm{N}_{\mathrm{eff}}=\mathrm{N}_{0} \exp (-c \phi)+\beta \phi
$$

For $n$-type devices such as ours, $\mathrm{N}_{0}$ is negative to indicate that it represents the initial number of (negative) donors. A fit of eq. (3) to the $27^{\circ} \mathrm{C}\left(0^{\circ} \mathrm{C}\right)$ data of Fig. 2 gives the constants $\beta=0.018(\beta=0.037) \mathrm{cm}^{-1}$ and $c=1.7 \times 10^{-1.3}$ $\left(c=1.1 \times 10^{-13}\right) \mathrm{cm}^{2}$. The relatively large values of both $\beta$ and $c$ indicate that the initial dopant concentration has only a minor effect on the effective dopant concentration for fluences of more than a few times $10^{13}$.

Type inversion, the change in sign of $\mathrm{N}_{\mathrm{eff}}$, occurs at room temperature at a fluence of approximately $10^{13} \mathrm{p} / \mathrm{cm}^{2}$. Since the sign of the dopant concentration does not enter into eq. 2 , type inversion has no immediate consequence for the operation of a detector, provided the detector is fully depleted. Thus, our interest in the change of the effective dopant concentration is only motivated by the need to bias the detectors beyond the depletion voltage at all times. (The voltage that can be applied is limited by the breakdown voltage of the detectors or their coupling capacitors.)

Short-term annealing of the radiation created acceptor states during the exposure and during the time between beam turn-off and the actual measurement can modify the values of $\beta, c$, and the fluence at which type reversal occurs. The high flux rates and short duration of the radiation exposure we used meant that the short-term annealing was incomplete. In contrast, the exposure at the SSC will involve low fluxes, but a high cumulative fluence. That will allow virtually all the shortterm annealing from the integral dose to be complete at any given time. As such, the values for the constants $\beta$ and $c$ given above will be over-estimates for the SSC application. For the same reason, type inversion will occur at higher cumulative doses. In addition, as shown below, temperature has a large effect on arnealing rates and will therefore affect the apparent values of those constants.

\section{Annealing}

We have reported before on the annealing of the radiation damage induced change of effective dopant concentration $[6,9]$ and found that the annealing can be the determining effect in that change. Figs. 3a, 3b show the post-irradiation time history of the depletion voltage for different annealing temperatures. All the devices shown received a fluence close to $5 \times 10^{13}$. Any small differences in fluence were accounted for by scaling the depletion voltages by the ratios of $5 \times 10^{13}$ to the fluences. This is justified by the proportionality of the depletion voltage to the fluence which is discussed later. The final annealing temperatures were implemented at different times as given in the figure caption.

The data exhibit a strong temperature dependence which is apparent in several ways. Fig. 3a shows that the beneficial annealing period (during which the depletion voltage drops) is very short for the room temperature device, but grows increasingly longer as the temperature is decreased. For the devices annealed at $21^{\circ} \mathrm{C}$ and $10^{\circ} \mathrm{C}$ a minimum value of the depletion voltage is reached, while the depletion voltage for the devices annealed at lower temperatures continued to decrease for the entire time period shown. Also note that the depletion voltage for the $-10^{\circ} \mathrm{C}$ device is well above the value for the $0^{\circ} \mathrm{C}$ device. At later times, following the beneficial annealing, the voltage begins to rise for the warmer devices in what we call anti-annealing. This is especially evident for the higher temperature devices as shown in Fig. $3 \mathrm{~b}$. The $35^{\circ}$ and $50^{\circ}$ data reach a constant depletion voltage value, which implies complete annealing, while the $24^{\circ}$ data do not. As the temperature is decreased, the time constant for the rise becomes progressively longer, and at $10^{\circ} \mathrm{C}$ becomes comparable with the SSC experiment lifetime ( $\sim 10$ years).

Based on the features in Fig. 3, we chose to describe the data in terms of several distinct steps governed by characteristic times. The short-term beneficial annealing period is assumed to be dominated by one time constant $\tau_{s}$ which describes the exponential decay of the active acceptor sites created during the irradiation period back to neutral inactive sites. We describe that region of the data at a time " $t$ " after the end of the irradiation by [6]:

$$
V_{D}(t)=V_{Z}+V_{S} \exp \left(-t / \tau_{S}\right)
$$

where $V_{S}$ is related to the metastable acceptor concentration directly produced during the irradiation process. $V_{\mathrm{Z}}$ is due to 

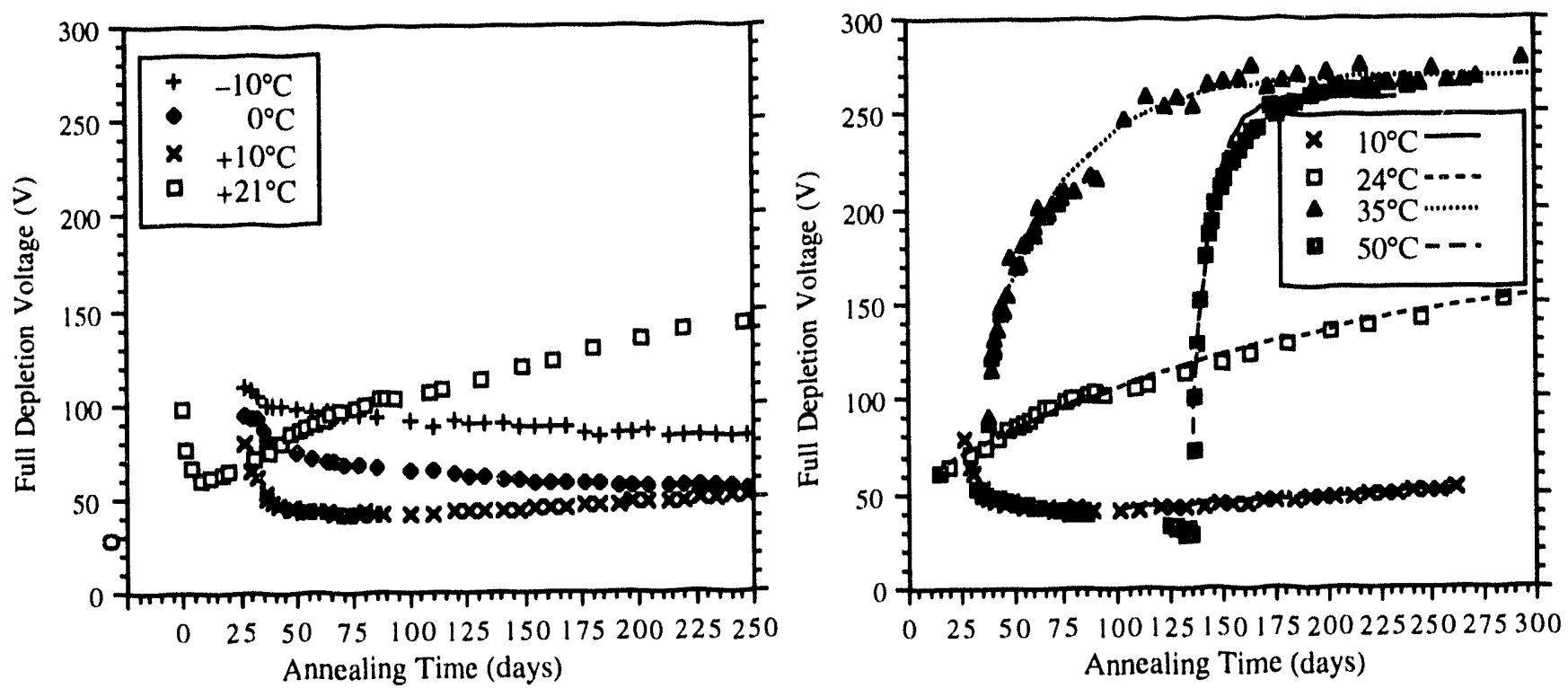

Figure 3): Depletion voltage as a function of time for deteciors being annealed at the indicated temperatures. All the devices shown rece to $5 \times 10^{13}$. Fits to the data of eq. $6\left(24^{\circ} \mathrm{C}, 35^{\circ} \mathrm{C}, 50^{\circ} \mathrm{C}\right)$ and eq. $7\left(+10^{\circ} \mathrm{C}\right)$ are shown. The final annealing temperatures were implemented as follows: $-10^{\circ} \mathrm{C}, 0^{\circ} \mathrm{C},+10^{\circ} \mathrm{C} @ 27$ days; $+35^{\circ} \mathrm{C} @ 36$ days; and $+50^{\circ} \mathrm{C} @ 137$ days. Prior to those times the temperature of the devices were as follows: the sub-ambient temperature devices: $\sim-20^{\circ} \mathrm{C}$ (some variation); the room temperature and $+35^{\circ} \mathrm{C}$ devices: ambient temperature; the $50^{\circ} \mathrm{C}$ devices: $\sim 0^{\circ} \mathrm{C}$.

the fraction of the radiation produced acceptor concentration which is stable. Therefore, $\mathrm{V}_{\mathrm{Z}}$ is the minimum value of the depletion voltage between the beneficial and anti-annealing regions.

The early time history (before day 25) of the sub-ambient temperature devices was not carefully controlled. Therefore, a determination of $V_{S}$ from the data for those devices is difficult. However, the room temperature devices were kept at a constant temperature during the early post-exposure period and thus should yield a reasonable estimate of $V_{S}$ provided their exposure period is not much longer than the time constant for beneficial annealing.

In contrast to the situation for $V_{S}$, if the beneficial annealing is described by eq. 4 , we should readily be able to estract the time constant $\tau_{S}$ from the data in the period after the temperature was stabilized, but before anti-annealing became comparable to the beneficial annealing. In Fig. 4 we plot the quantity $\left[\ln \left(\frac{V(t)-V_{2}}{V_{0}}\right)\right]$ as a function of the annealing time. The depletion voltage, when the temperature was stabilized, is $V_{0}$. $\mathrm{V}_{\mathrm{Z}}$ is given by the minimum value of the depletion voltage. (For the $-10^{\circ} \mathrm{C}$ data we used the results from eq. 8 a below, and the known fluence to estimate the value of $\mathrm{V}_{\mathrm{Z}}$.) If eq. 4 does describe the data, then a straight line fit through the data points for a given temperature data set should have a slope of $\left(-\tau_{S}\right)^{-1}$. The average values found at the different temperatures are given in Table 1. In Fig. 5 we show a semilog plot of $\tau_{s}$ as a function of temperature. A fit of eq. 5 to the data yields:

$$
\tau_{S}(\mathrm{~T})=70 \times \exp (0.175 \mathrm{~T}) \text { days; } \mathrm{T} \text { in }{ }^{\circ} \mathrm{C}
$$

The results for the $21^{\circ} \mathrm{C}$ data yield a value of 1.8 days for $\tau_{\mathrm{S}}\left(21^{\circ} \mathrm{C}\right)$. We can now derive a value for $\mathrm{Vs}$ from four $21^{\circ} \mathrm{C}$ devices, all of which received a fluence of $7.0 \times 10^{13} \mathrm{p} / \mathrm{cm}^{2}$ over two days. Those devices were first measured three hours after the beam was turned off. Their average depletion voltage at that time was $139 \mathrm{~V}$. Their depletion voltage decreased over the next several days to an average minimum value of $86.7 \mathrm{~V}$. Taking the difference of those two values and using eq. 4 to extrapolate back 3 hours in time to the end of the exposure, we find $V_{S}(e n d)=56.5 \mathrm{~V}$. Assuming the beam flux was uniform over the two day exposure, an additional correction factor of 1.66 is needed to account for decays during the exposure, yielding 94.0 $\mathrm{V}^{\dagger \dagger}$ as the value of $\mathrm{V}_{\mathrm{S}}$ for an instantaneous exposure. Taking $V_{S}=v_{s} \phi$, we finally find $v_{S}=1.34 \times 10^{-12} \mathrm{~V}-\mathrm{cm}^{2}, \phi$ being the fluence.

At larger times, the depletion voltage increases (anti-anneals). Fig. 3b shows that the rate of anti-annealing slows with time for the devices at $+24^{\circ} \mathrm{C},+35^{\circ} \mathrm{C}$, and $+50^{\circ} \mathrm{C}$. We fit the time dependence of the depletion voltage for this period with the function:

$$
V_{D}(t)=V_{Z}+V_{A}\left[1-\exp \left(-t / \tau_{L}\right)\right] ; \quad t>\tau_{S}
$$

where $\tau_{1}$ is the long-term anti-annealing time constant, $t$ is measured from the time at which the anti-annealing first becomes evident, and $V_{A}$ is related to the concentration of damage sites in silicon that can become activated acceptor sites

It Attempts to extract $V_{S}$ from the lower temperature devices, whose early annealing temperatures were not carefully controlled, gave widely varying results, some comparable to the $21^{\circ} \mathrm{C}$ values, but others considerably smaller. 


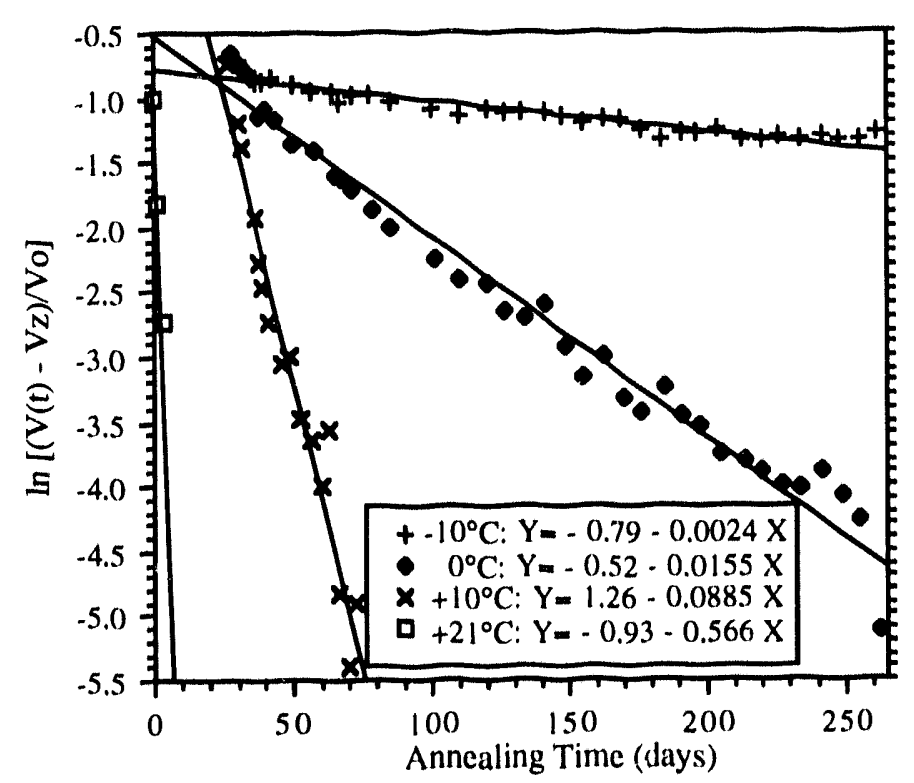

Figure 4): A plot of $\ln \left(\frac{V(t)-V_{Z}}{V_{0}}\right)$ as a function of the annealing time. $V_{0}$ is the depletion voltage, when the temperature was stabilized. $V_{Z}$ is given by the minimum value of the depletion voltage. (For the $-10^{\circ} \mathrm{C}$ data we used the results from eq. $8 \mathrm{a}$, and the known fluence to estimate the value of $\mathrm{V}_{\mathrm{Z}}$.) Straight line fits through the data are also shown. Their slope should be $\left(-\tau_{\mathrm{S}}\right)^{-1}$.

due to anti-annealing. The data at $+50^{\circ},+35^{\circ}$ and $+24^{\circ} \mathrm{C}$ allow us to fit for $V_{Z}, V_{A}$, and $\tau_{L}$ simultaneously. At lower temperatures the exponential behavior of the anti-annealing process is not very obvious as can be seen for the $10^{\circ} \mathrm{C}$ data. The effective expansion of the time scale caused by lower temperatures makes the anti-annealing appear to be a linear function of time, as given by a first order Taylor series expansion of eq. 6 :

$$
V_{D}(t)=V_{Z}+V_{A} t / \tau_{L} ; \quad t \ll \tau_{l}
$$

We fit eq. 7 to both the data for the $+10^{\circ} \mathrm{C}$ devices irradiated in 1992 and the $0^{\circ} \mathrm{C}$ devices from 1991 to extract $V_{A} / \tau_{1}$. Using values for $\mathrm{V}_{\mathrm{A}}$ derived from the combined $35^{\circ} \mathrm{C}$ and $50^{\circ} \mathrm{C}$ data set and the known fluences, we are able to find $\tau_{L}$ for the low temperature devices.

The values of the constants derived from those fits are shown in Figs. 6, 7, 8 and are given in Table $1^{+1 t}$. Fig. 6 shows the values for the non-annealing voltage $V_{Z}$ as function of fluence. An approximate linear dependence on the fluence is seen with no obvious temperature dependence. We fit the combined data for all temperatures for which the short-term annealing was complete $\left(+10^{\circ}, 24^{\circ}, 35^{\circ}\right.$, and $\left.50^{\circ}\right)$ as a linear function of the fluence $\phi$ :

$$
v_{Z}(\phi)=v_{Z} \phi ; \quad v_{Z}=1.06 \times 10^{-12}
$$

Itt All the results for $V_{X}$ and $v_{X}$ scale as the square of the detector thickness ( $f$. eq. 2 ). The values given in this paper are for $300 \mu \mathrm{m}$ thick detectors. (X $=S, Z, A, O R U)$

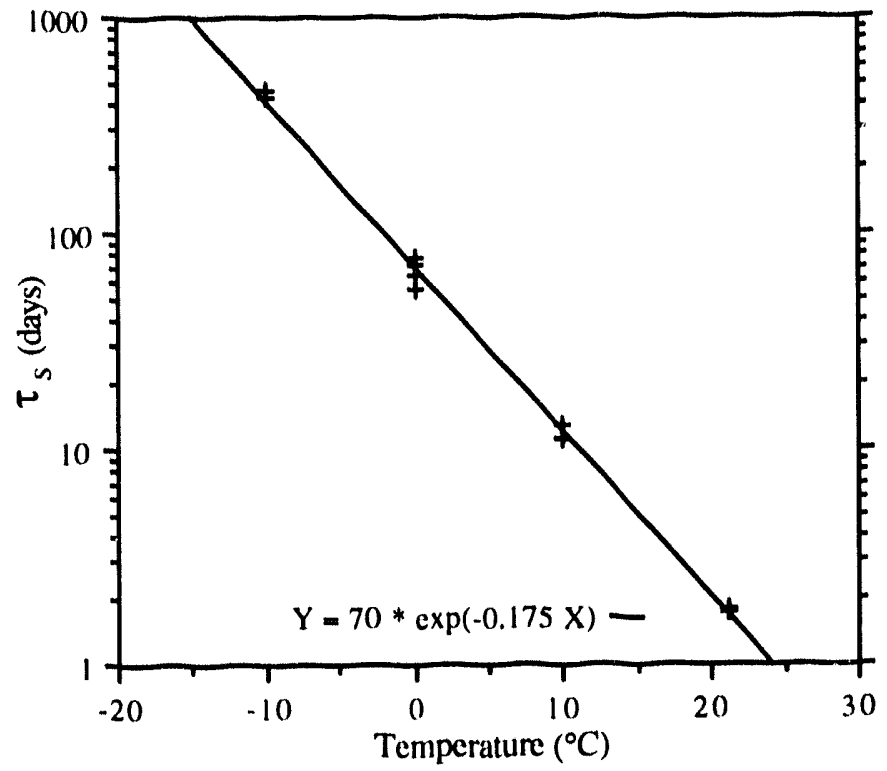

Figure 5): The beneficial annealing time constants $\tau_{S}$ as a function of annealing temperature in ${ }^{\circ} \mathrm{C}$. The fit shown gives $\tau_{S}=70 \times \exp (-0.175 \mathrm{~T})$ days.

Fig. 7 shows the values for the anti-annealing voltage $V_{A}$ as function of fluence. An approximate linear dependence on the fluence is seen. The line in Fig. 7 shows a fit to the combined $35^{\circ} \mathrm{C}$ and $50^{\circ} \mathrm{C}$ data sets as a function of fluence $(\phi)$ :

$$
v_{A}(\phi)=v_{A} \phi ; \quad v_{A}=3.80 \times 10^{-12}
$$

In Fig. 7 , the data for $24^{\circ} \mathrm{C}$ are systematically lower than the data for $35^{\circ} \mathrm{C}$ and $50^{\circ} \mathrm{C}$ and were not included in the fit. The time dependence of the anti-annealing might be somewhat more complicated than indicated in eq. 6, possibly involving a second, slightly different, time constant. That is supported by the imperfect nature of the fits we obtain as evidenced in Fig. 3b. The data seem to support a time constant that is slightly smaller at early times than at later times. Thus fitting only the first part of the annealing curve, as for the $24^{\circ} \mathrm{C}$ data, leads to an underestimate of the final anti-annealing voltage. On the other hand, the observed saturation of the $35^{\circ} \mathrm{C}$ and $50^{\circ} \mathrm{C}$ data give us confidence that eq. 6 predicts the long-term behavior.

TABLE 1: The values of $v_{S}, v_{Z}, v_{A}, v_{U}$, and the values of $\tau_{S}$ and $\tau_{1}$, for the different temperatures and for all temperatures.

\begin{tabular}{|c|c|c|c|c|c|}
\hline & & $T$ & $\tau_{S}$ & $T$ & $\tau_{L}$ \\
\hline & $\left(V-\mathrm{cm}^{2}\right)$ & $\left({ }^{\circ} \mathrm{C}\right)$ & $($ days $)$ & $\left.{ }^{\circ} \mathrm{C}\right)$ & $($ days $)$ \\
\hline$v_{S}$ & $1.34 \times 10^{-12}$ & -10 & 440 & -10 & $?$ \\
\hline$v_{Z}$ & $1.06 \times 10^{-12}$ & 0 & 66.7 & 0 & 8650 \\
\hline$v_{A}$ & $3.80 \times 10^{-12}$ & 10 & 12.1 & 10 & 3320 \\
\hline$v_{U}$ & $4.78 \times 10^{-12}$ & 21 & 1.8 & 24 & 186 \\
\hline & & $?$ & 35 & 38 \\
\hline 35 & $?$ & 50 & 12 \\
\hline 50 & all & $\begin{array}{c}7() \times \mathrm{exp} \\
(-0.175 \mathrm{~T})\end{array}$ & all & $\begin{array}{c}9140 \times \mathrm{exp} \\
(-0.152 \mathrm{~T})\end{array}$ \\
\hline
\end{tabular}




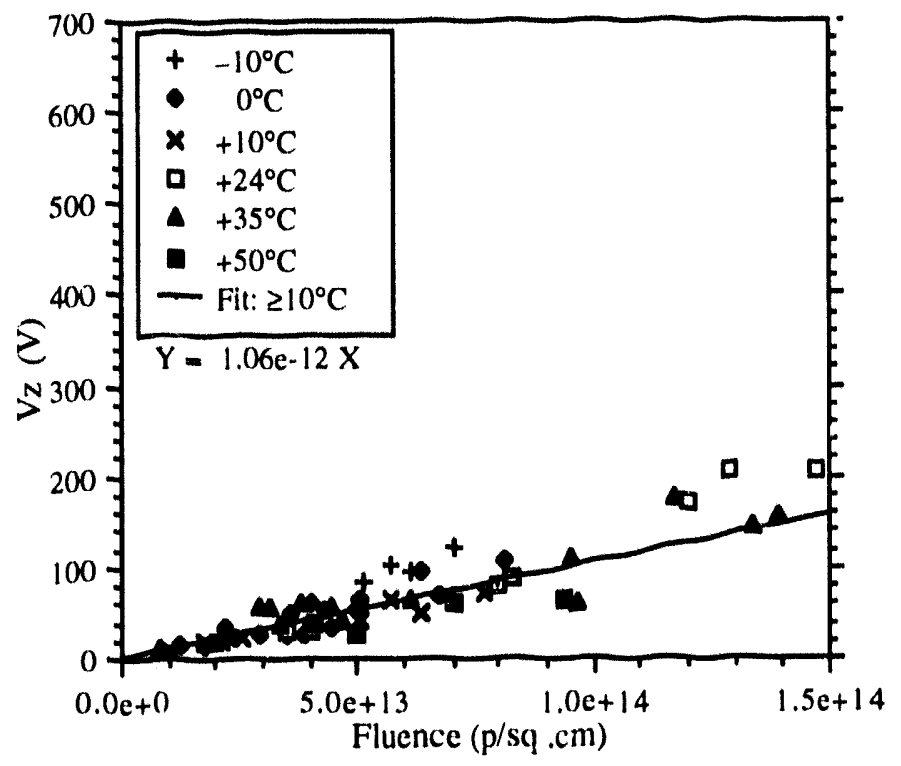

Figure 6): The minimurn value of the depletion voltage $\left(V_{Z}\right)$ reached, after short-term annealirg, as function of fluence. The different temperature detectors are indicated. For the devices kept at $0^{\circ} \mathrm{C}$ and $-10^{\circ} \mathrm{C}$ the values are upper limits as the depletion voltage did not reach a minimum value. A fit of the form $V_{Z}=v_{Z} \phi$ to the $210^{\circ} \mathrm{C}$ data is indicated.

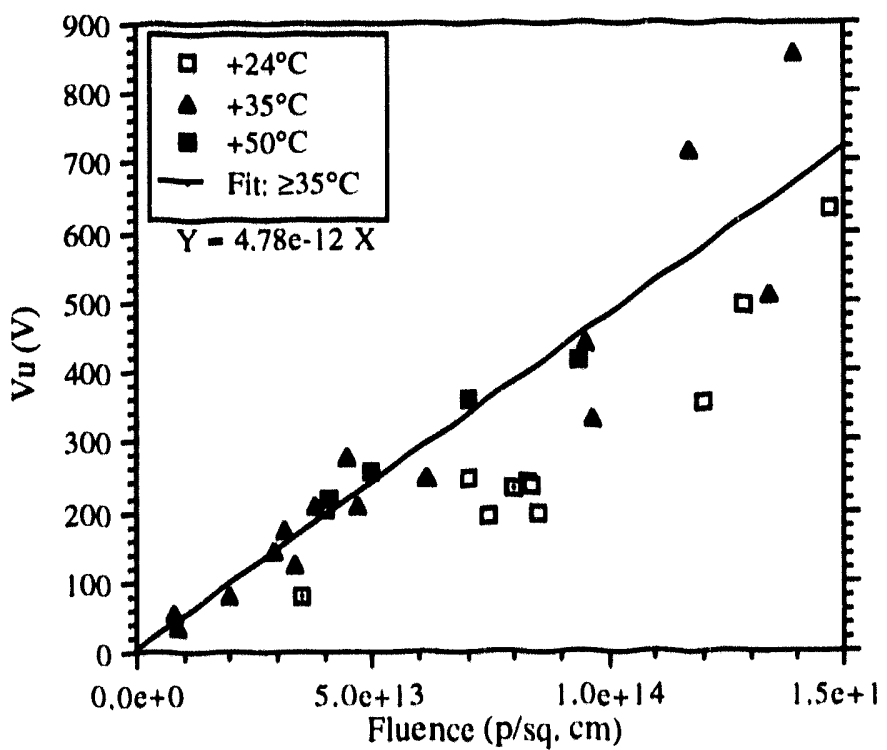

Figure 8): The ultimate depletion voltage after annealing $\left(V_{U}-V_{Z}+V_{A}\right)$, as function of fluence. A lincar fit to the combined $35^{\circ} \mathrm{C}$ and $50^{\circ} \mathrm{C}$ temperature sets is indicated. Note the reduced scatter of the data when compared with Figs. 6 and 7.

The values for $\mathrm{V}_{\mathrm{Z}}$ and $\mathrm{V}_{\mathrm{A}}$ are correlated: overestimating $\mathrm{V}_{\mathrm{Z}}$. leads to a smaller value for $\mathrm{V}_{\mathrm{A}}$ and vice versa. Their sum should be more stable. Fig. 8 shows the values for the ultimate depletion voltage $V_{U}-V_{Z}+V_{A}$ after annealing and anti-annealing. A linear dependence on fluence is found:

$$
V_{U}(\phi)=V_{Z}(\phi)+V_{A}(\phi)=V_{U} \phi ; \quad V_{U}=4.78 \times 10^{-12}
$$

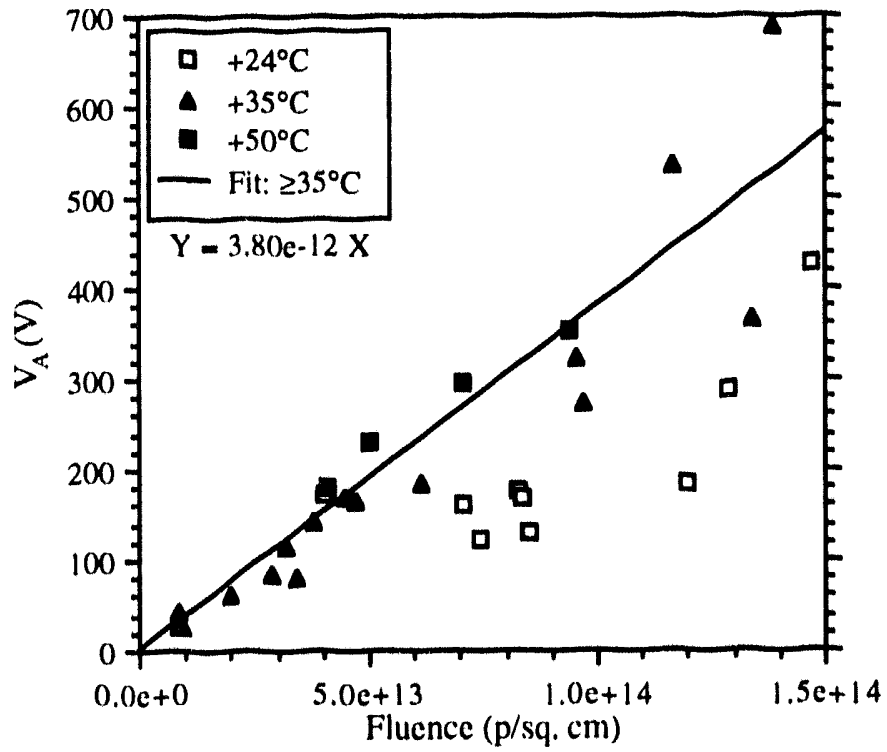

Figure 7): The anti-annealing voltage $V_{A}$ as function of fluence. $A$ linear fit to the combined $35^{\circ} \mathrm{C}$ and $50^{\circ} \mathrm{C}$ temperature data sets is indicated. Note the difference in the magnitude of $\mathrm{V}_{\mathrm{A}}$ and $\mathrm{V}_{\mathrm{Z}}$.

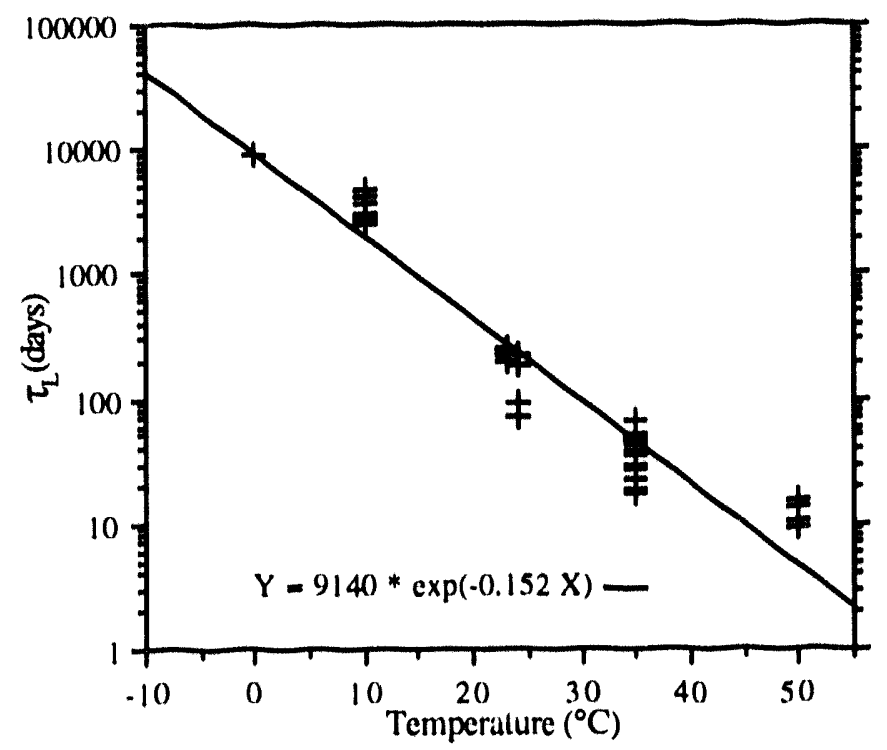

Figure 9): The anti-annealing time constants $\tau_{l}$ for all detectors as a function of annealing temperature in ${ }^{\circ} \mathrm{C}$. The fit shown gives $\tau_{I_{1}}-9140 \times \exp (-0.152 \mathrm{~T})$ days.

Fig. 9 shows the values of the long anti-annealing time constant $\tau_{L}$ as a function of temperature for all the devices except those at $-10^{\circ} \mathrm{C}$, for which it was too large to be determined. The average value of $\tau_{1}$ for each temperature is given in Table 1. The temperature dependence as shown in Fig. 9 is of the form: 


$$
\tau_{\mathrm{l}}(\mathrm{T})=9140 \times \exp (0.152 \mathrm{~T}) \text { days; } \quad \mathrm{T} \text { in }{ }^{\circ} \mathrm{C}
$$

- We do not observe a fluence dependence in $\tau_{1 .}$. The values of $\tau_{\mathrm{L}}$ for $0^{\circ} \mathrm{C}, 10^{\circ} \mathrm{C}$ and $24^{\circ} \mathrm{C}$ are at least a factor $1(0)$ larger than the corresponding values of $\tau_{\mathrm{s}}$.

\section{Predictions}

We are now in the position to make definite predictions about the long-term dependence of the depletion voltage on fluence and temperature. Important parts of these predictions are the observations made above and summarized here:

i) The data are described by eqs. 4 and 6;

ii) $V_{S}, V_{2}$, and $V_{A}$ are independent of temperature;

iii) $V_{S}, V_{z}$, and $V_{A}$ depend linearly on the fluence (cf. eq.8);

iv) $\tau_{S} \ll \tau_{1}$;

v) The annealing time constants $\tau_{S}$ and $\tau_{L}$ are exponential functions of the temperature;

vi) The annealing time constants $\tau_{s}$ and $\tau_{1}$, are independent of the fluence.

The change in depletion voltage due to an instantaneous fluence $\phi$ delivered at time $\mathrm{t}_{0}$ is given by: $\left(\Delta \mathrm{t}=\mathrm{t}-\mathrm{t}_{0}\right)$

$v_{D}(\Delta t)=\phi\left\{v_{\gamma}+v_{s} \exp \left(-\Delta t / \tau_{S}\right)+v_{A}\left(1-\exp \left(-\Delta t / \tau_{L}\right) \mid\right\}\right.$

The temperature dependence is in the annealing time constants (eqs. 5, 9). If one includes the effects of a continuous flux $F$ starting at $\mathrm{t}=0$, then eq. 10 generalizes to:

$$
\begin{aligned}
v_{D}(t)= & v_{Z} F t+ \\
& v_{S} F \tau_{S}\left|1-\exp \left(-v / \tau_{\cdot}\right)\right|+ \\
& \left.v_{A} F\left\{t+\tau_{1} \mid-1+\exp \left(-t / \tau_{1}\right)\right\}\right\}
\end{aligned}
$$

We have used eq. 11 to predict the rise in depletion voltage as a function of time assuming a constant SSC flux of $1.3 \times 10^{13} \mathrm{p} / \mathrm{cm}^{2}-y r$. Clearly, our model does not explain the early complicated behavior up to type reversal. Rather, it addresses the ultimate depletion voltage after 10 years of exposure to a total of $1.3 \times 10^{14}$ particles $/ \mathrm{cm}^{2}$, which is of interest to us. Using the values for $v$ given in Table 1 and the value of $\tau_{\mathrm{S}}$ and $\tau_{1}$. predicted by eqs. 5 and 9 respectively, in Fig. 10) we show the cumulative effect on the depletion voltage of a flux of $1.3 \times 10^{13} \mathrm{p} / \mathrm{cm}^{2}$ per year. The curves are for different operating (and annealing) temperatures. Mindful of eq. 2, we propose to use detectors $250 \mu \mathrm{m}$ thick instead of the standard $30 x) \mu \mathrm{m}$ thickness, which reduces the depletion voltage by $30 \%$. Preliminary studies showed that detectors of 50 $\mu \mathrm{m}$ pitch operated in a magnetic field will have no degraded signal/noise when thinned from $3(0)$ to $250 \mu \mathrm{m}$ [10].

Eq. 11 allows us to evaluate the optimal operating temperature of the silicon detectors at the SSC. The $v$, term in eq. 11 contains no elements which have a temperature dependence. Rather it is directly proportional to the fluence and hence increases linearly with the running time. In contrast, the term containing $v_{S}$ has no long-term time dependence, reaching a saturation value of $v_{S} F \tau_{S}$. The magnitude of the saturation value can be controlled through the temperature dependence of $\tau_{s}$. For a flux of $10^{13} \mathrm{p} / \mathrm{cm}^{2}-\mathrm{yr}$, the saturation value is $19 \mathrm{~V}$

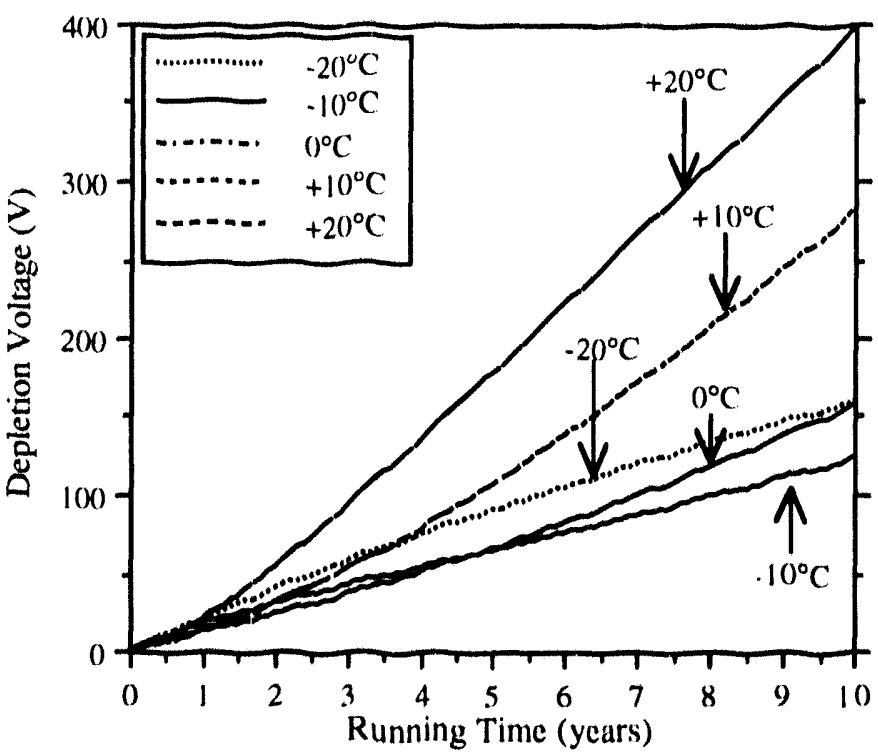

Figure 10): Predictions of the change in the depletion voltage for a 250 micron thick detector over 10 years of SSC operation for different detector temperatures. A uniform yearly fluence of $1.3 \times 10^{1.3} \mathrm{p} / \mathrm{cm}^{2}$ was assumed.

for $-10^{\circ} \mathrm{C}$, but drops to only $3 \mathrm{~V}$ at $0^{\circ} \mathrm{C}$. As can be seen, the term only becomes important if the temperature is low enough to substantially slow the beneficial annealing through an increase in $\tau_{s}$. Smaller values of $v_{s}{ }^{\dagger+}$ would make this term even less important. Finally, the term containing $v_{A}$ is potentially the most important, given that $\nu_{\mathrm{A}}$ is about three times larger than $v_{Z}$ or $v_{S}$, and that the term at large times can be nearly proportional to the fluence. However, the term's contribution is strongly controlled through the value of $\tau_{1}$, which in turn is determined by the temperature. By making $\tau_{1}$. sufficiently large compared to the integral exposure time to the radiation, the term becomes negligible. In summary, an operating temperature should be chosen, which allows beneficial annealing to oceur, but at the same time totally suppresses the anti-annealing. In that case, ones get the minimum detector depletion voltage and hence the maximum detector lifetime; the lifetime now only determined by the breakdown voltage of the detector, the value of $v_{\%}$, and the integral fluence to which the detector was exposed.

Fig. 10 shows that the optimal operating temperature of the silicon detectors at the $\mathrm{SSC}$ is $\sim-10^{\circ} \mathrm{C}$. After a fluence of $1.3 \times 10^{14} \mathrm{p} / \mathrm{cm}^{2}$, we predict a depletion voltage of about 12.5 (160) $V$ for detectors $250 \mu \mathrm{m}$ thick and operated at a temperature of $-10^{\circ}\left(0^{\circ}\right) \mathrm{C}$. Collecting the charge promptly requires a somewhat greater total applied voltage $(150-200 \mathrm{~V})$. Double-sided AC coupled detectors could be operated at this depletion voltage if biased on the junction and ohmic sides at $\pm 1(0) \vee(111$. If one assumes a detector breakdown voltage of $200 \mathrm{~V}$, and a required overvoltage of $40 \mathrm{~V}$ for prompt signal collection, a detector operated at $00^{\circ} \mathrm{C}$ would just have a 10 -year lifetime. Operating the same detector at $+10^{\circ} \mathrm{C}$ would reduce 
the useful life to 6.6 years and keeping it at $+20^{\circ} \mathrm{C}$ reduces the lifetime to 4.6 years.

An importint consideration for operation at sub-ambient temperature is how long the detectors can be warmed up for service and repairs. Our analysis shows that warming will predominately influence the anti-annealing. To first order the effects will depend on the value of the anti-annealing time constant $\tau_{\mathrm{L}}$ at the new, warmer temperature. From eq. 7 follows:

$$
\Delta V_{D}=V_{A} \Delta t / \tau_{L}
$$

Using eq. 8b, and inverting eq. 12, one can so!ve for the amount of time $(\Delta t)$ that a detector can be warmed, after having been operated for " $t$ " years at a constant flux F, to induce a change in the depletion voltage of $\Delta V_{D}{ }^{+1+\dagger}$.

$$
\Delta t=\frac{\Delta V_{D} \tau_{L}(T)}{F t v_{A}}
$$

The allowable duration of an elevated temperature $T$, for a 4-volt increase in the depletion voltage (i.e. a $2.5 \%$ change in an ultimate depletion voltage of $160 \mathrm{~V}$ ) is given in Table 2 and ranges from 8 months at $+10^{\circ} \mathrm{C}$, to under one month at $25^{\circ} \mathrm{C}$ in the first year of SSC running. After 10 years of SSC running, the duration ranges from several weeks at $+10^{\circ} \mathrm{C}$ to only a couple of days at $25^{\circ} \mathrm{C}$. With this model we can easily evaluate other scenarios of the fluence profile over the lifetime of the SSC.

Strictly speaking, our measurements and the parameters in our fits apply only to damage due to protons. Ref. [2] predicts that pions, which make up most of the fluence at the SSC, have damage factors about $1 / 2$ of that of protons. Until this is confirmed, we will use the possible lower depletion due to pion irradiation as one of the SSC contingencies.

TABLE 2: The allowable duration $\Delta t$ (in days) of a warm-up to a temperature $T$, which causes a $4 \mathrm{~V}$ increase in the depletion voltage assuming a constant flux of $1.3 \times 10^{13} \mathrm{p} / \mathrm{cm}^{2}-\mathrm{yr}$. It is given for warm-up at different elapsed times of SSC operation in years.

\begin{tabular}{|c||c|c|c|c|}
\hline \multicolumn{1}{|c||}{} & \multicolumn{4}{|c|}{ SSC Operation Time } \\
\hline $\left.\mathrm{T} 1^{\circ} \mathrm{C}\right)$ & 1 year & 2 years & 5 years & 10 years \\
\hline 10 & 234 & 117 & 47 & 23 \\
\hline 15 & 110 & 55 & 22 & 11 \\
\hline 20 & 51 & 26 & 10 & 5 \\
\hline 25 & 24 & 12 & 5 & 2 \\
\hline
\end{tabular}

tttt For a $250 \mu \mathrm{m}$ thick detecdtor, $v_{A}$ must be reduced $t+1$ by $30 \%$ from the eq. $8 \mathrm{~b}$ value.

\section{CONCLUSION}

Based on measurements of the depletion voltage during irradiation and annealing at different temperatures, we have developed a model which describes the fluence, time, and temperature dependence of the change in depletion voltage in terms of a few measurable parameters. We are able to predict the depletion voltage during long-term operations at the SSC. The critical effect is the temperature dependence of the longterm anti-annealing. A judicious choice of the operating temperature $\left(\sim 0^{\circ} \mathrm{C}\right.$ to $\left.-20^{\circ} \mathrm{C}\right)$, yields benefits from the shortterm annealing, which lowers the depletion voltage caused by the radiation induced displacement damage. At the same time, the long-term detrimental anti- or reverse annealing effects can be frozen out for periods longer than the expected 10-year life of the experiments at the SSC. The net damage is consistent with a 10-year operational life of the detectors, which corresponds to a fluence of slightly above $10^{14}$ minimum ionizing hadrons $/ \mathrm{cm}^{2}$. We also predict the allowable duration of service time at elevated temperatures at varying times of SSC operation. That duration, ranging from months to only days, is found to depend on both the integral fluence up to the time of the service and the temperature at which the service is performed.

\section{REFERENCES}

1) "Calculation of Neutron Backgrounds and the Production and Decay of Radionuclides in the SDC Detector," A.P.T. Palounck, L.S. Waters, T.R. England, H.G. Hughes, H. Lichtenstein, R.E. Pracl, W.B. Wilson, and H.J. Ziock, Los Alamos National Laboratory Report LA-UR-93-1217.

(2) "Non lonizing Energy Deposition in Silicon for Radiation Damage Studies", A. Van Ginneken, FNAL preprint FN-522, Oct 1989.

[3] "Radiation Damage in Silicon Detectors", H.W. Kramer, NIM 225 p615.(1984);

"Radiation Damage in Silicon Strip Detectors", H. Dietl, T, Gooch, R.KInner, M.Cope, F.D. Wichen, NIM A253, 460 (1987);

"Measurement of Proton Induced Radiation Damage to CMOS Transistors and PIN Diodes", H.J. Ziock, C.M. Hoffman, D. Holtkamp, W.W. Kinnison, C. Milner, W.F. Sommer, J. Bacigalupi, N. Cartiglia, J. DeWitt, A. Kaluzniacki, H. Kolanoski, D. Pitzl, W.A. Rowe, H.F.W. Sadrozinski, E. Spencer, P. Tennenbaum, P. Ferguson, E.C. Milner, W.F. Sommer, P. Giubcllino, and S. Sartori, IEEE Transactions on Nuclear Science, Vol 37, No. 3, (June 1990).

14] D. Dorfan, These proceedings.

(5) "Physics of Semiconductor Devices", S.M. Sze, New York, Wiley, 1981, p. 90.

[6] "Temperature Dependence of Radiation Damage and its Annealing in Silicon Detectors", H.J.Ziock, J.G. Boissevain, K. Holzscheiter, J.S. Kapustinsky, A.P.T. Palounek, and W.E. Sondheim, E. Barberis, N. Cartiglia, J, Leslie, D. Pitzl, W.A. Rowe, H.F.--W. Sadrozinski, A. Seiden, E. Spencer, M. Wilder, J.A. Ellison, J.K. Fleming, S. Jerger, D. Joyee, C. Lietzke, E. Reed, and S.J. Wimpenny, P. Ferguson, M.A. Frautschi, 
J.A.J. Matthews, and D.Skinner, IEEE Transactions on Nuclear Science, August 1993, Vol. 40), Number 4, p.344348.

[7] Hamamatsu Photonics K.K, Ichino-cho, Hamamatsu, Japan

[8] Micron Semiconductors Ltd., Lancing, Sussex, UK

19] "Type Inversion in Silicon Detectors", D. Pitzl, N. Cartiglia, B. Hubbard, D. Hutchinson, J. Leslie, K. O'Shaughnessy, W. Rowe, H. F. W. Sadrozinski, A. Seiden, E. Spencer (UC, Santa Cruz), H.-J. Zlock, P. Ferguson, K. Holzscheiter, and W. F. Sommer (Los Alamos), Published in NIM A3H (Jan. 1992) 98-104.

[10] A. Seiden, IEEE Nucl. Sci. Symp., San Francisco, Nov 1993.

[11] A. Weinstein et al.,"Silicon Tracker Conceptual Design Report Nov 1992, SCIPP 92-(1)4. 

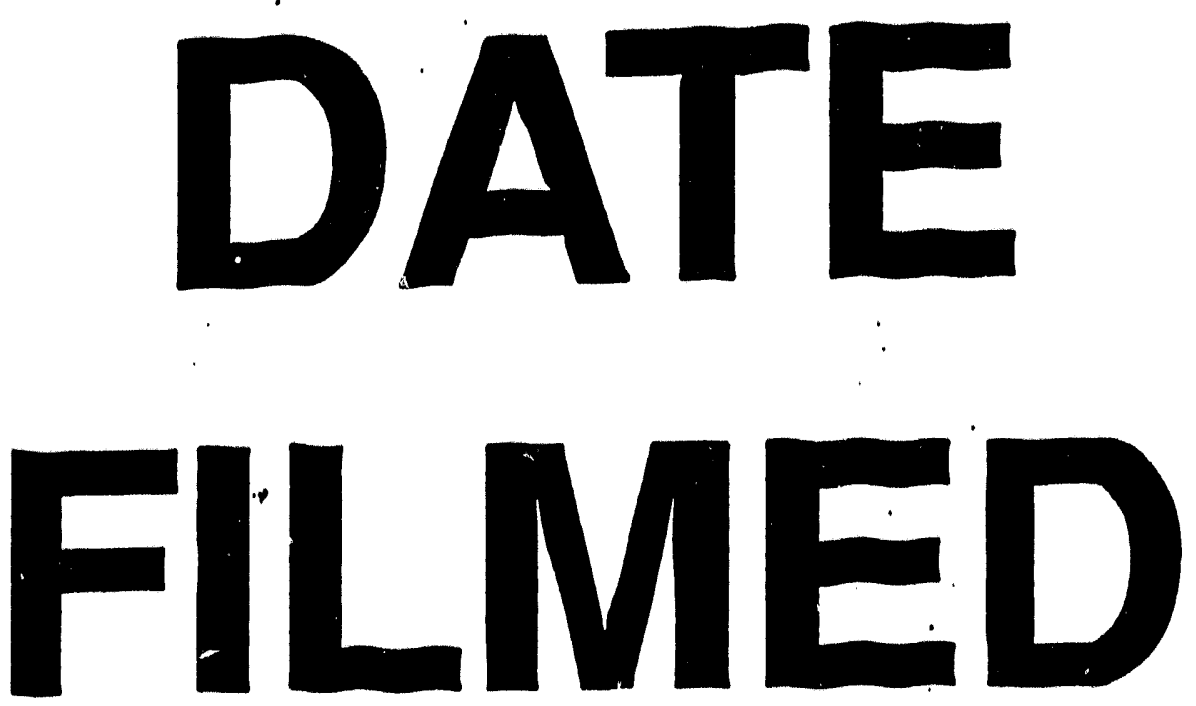

1
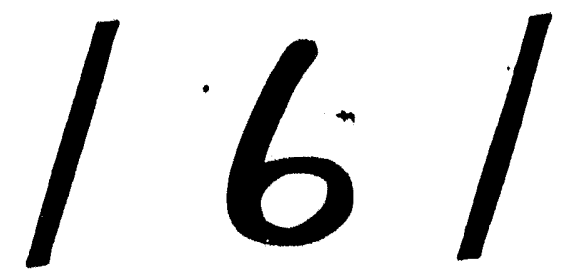

94
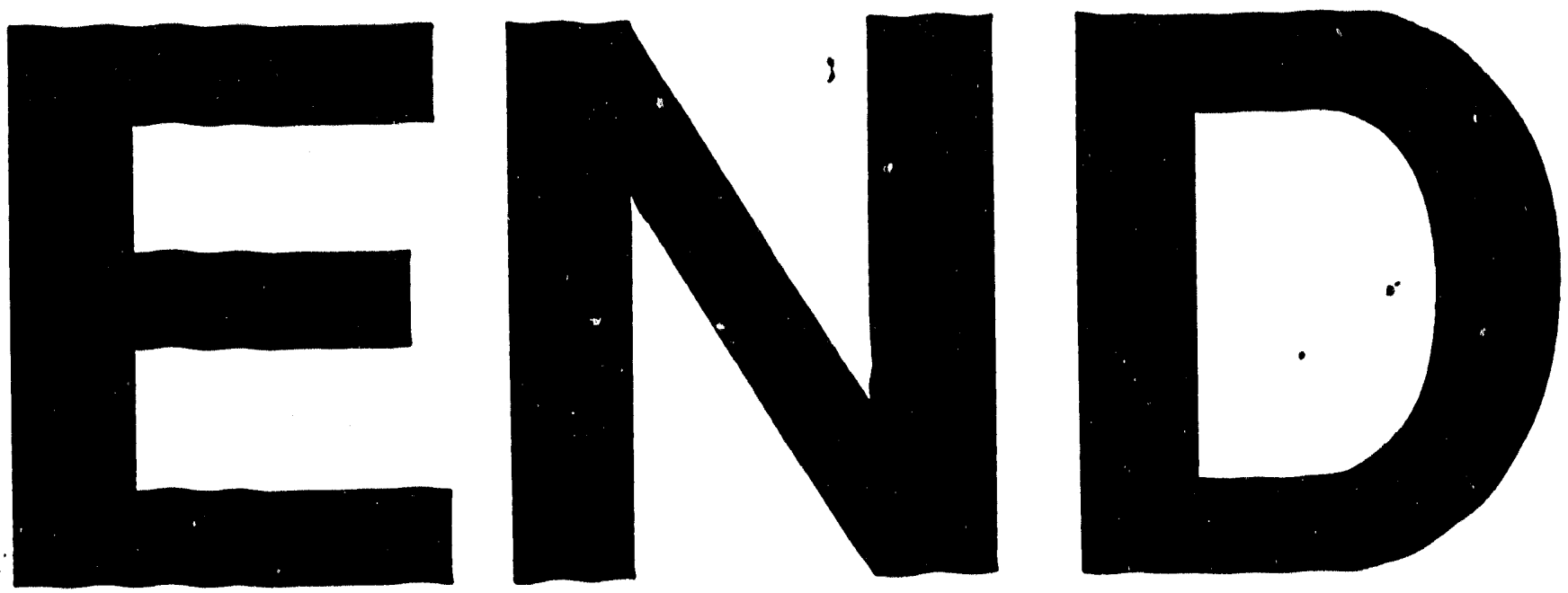


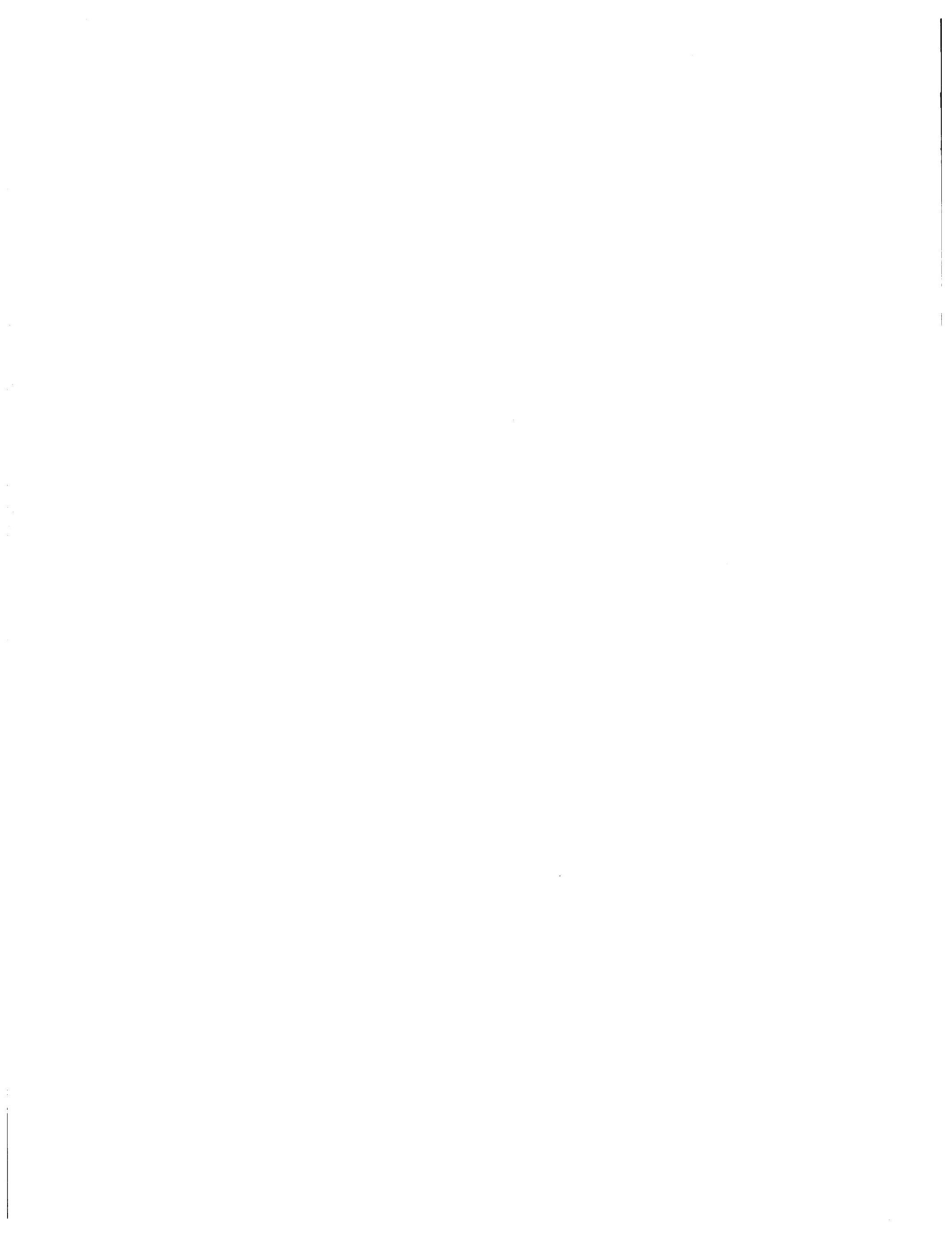

